\title{
Synthesis of carbohydrate-based antibiotics*
}

\author{
Chi-Huey Wong ${ }^{\ddagger}$, Marian C. Bryan, Paul T. Nyffeler, Haitian Liu, and \\ Eli Chapman
}

\author{
Department of Chemistry, The Scripps Research Institute, 10550 N. Torrey Pines \\ Road, La Jolla, CA 9203, USA
}

\begin{abstract}
Tackling the problem of carbohydrate recognition is a subject of current interest. Our strategies in this regard include development of new methods for the synthesis of saccharides and sugar arrays for screening, design of D-peptides to target bacterial cell-surface sugars, preparation of mechanism-based inhibitors of the enzymes involved in the biosynthesis of saccharides and the synthesis of aminoglycoside mimetics to target RNA.
\end{abstract}

\section{INTRODUCTION}

The chemistry and biology of carbohydrates is an emerging field of interest, especially in this postgenomic era. Carbohydrate modifications of proteins and lipids are important processes that modulate the structures and functions of these biomolecules and affect intercellular recognition in infection, cancer, and immune response. The mechanisms of these events are, however, not well understood, and most recent efforts in the field are to develop new tools for use to understand the molecular-level carbohydrate recognition and to enable the carbohydrate-based drug discovery process.

The emergence of antibiotic resistance is a growing problem in the treatment of infectious diseases [1]. To date, even vancomycin, the last resort of antibiotics active against staphylococcus aureus and some other gram-positive bacteria, has encountered resistance [2], and this new public health crisis has renewed the interest in antibacterial development [3].

Three mechanisms are commonly found in antibiotic resistance: first, the antibiotic is pumped out by the bacterial efflux pump on the cell wall; second, the antibiotic is modified by the bacterial enzymes to weaken its interaction with the target; third, the antibiotic target is modified in the bacterial genome. Current strategies for tackling the problem of antibiotic resistance generally involve chemical modification of existing antibiotics to resist these mechanisms or development of new types of antibiotics. Our interest in the field is to develop new antibiotics that target unique carbohydrates or carbohydrate-utilizing enzymes in bacteria and resist the resistance development.

\section{D-PEPTIDES THAT TARGET BACTERIAL CELL-SURFACE CARBOHYDRATES}

The cell surface of bacteria often contains unique carbohydrates such as 3-deoxy-D-manno-2-octulosonic acid (KDO) and heptoses. Development of small D-peptides that are stable in the circulation and bind the bacterial cell-surface sugar with high affinity may have potential as new antibiotics. Our approach to the discovery of such enantiomeric oligomers is to use the phage display method to identify L-peptides that bind the enantiomer of the cell-surface sugar (i.e., L-sugars).

\footnotetext{
* Pure Appl. Chem. 75, 141-419 (2003). An issue of reviews and research papers based on lectures presented at the $23^{\text {rd }}$ IUPAC International Symposium on the Chemistry of Natural Products, Florence, Italy, 28 July-2 August 2002 on the theme of natural products.

${ }^{\ddagger}$ Corresponding author
} 
Once the high-affinity oligomers are identified, their enantiomers (i.e., D-peptides) are synthesized chemically and used to target the natural cell-surface sugars. We have developed a chemoenzymatic synthesis strategy for the preparation of several L-sugars, including, for example, L-KDO, L-sialic acid, L-3-deoxy-D-glycero-D-galacto-2-nonulosonic acid (KDN), L-galactose, L-galactosamine, L-glucose, and L-glucosamine, used as building blocks for the synthesis of L-oligosaccharides. These enantiomeric carbohydrates have been used as affinity ligands for the identification of high-affinity peptides that recognize L-KDO and L-sialic acid as an effort to develop a general strategy for use to develop Dpeptides to target cell-surface carbohydrates [4].

\section{INHIBITORS OF CARBOHYDRATE AND PEPTIDOGLYCAN BIOSYNTHESIS}

The peptidoglycan biosynthesis pathway has been a target for antibiotic development, and most existing antibiotics, such as vancomycin and $\beta$-lactam antibiotics, target the transpeptidase-catalyzed crosslinking step of this pathway. Resistance to the transpeptidase inhibitors is often developed through modification of the peptide moiety to weaken the antibiotic potency or through the action of $\beta$-lactamase to inactivate the antibiotics [2,5]. One way to tackle the problem of vancomycin resistance is to use polyvalent vancomycin with higher affinity for the wild-type D-Ala-D-Ala and the resistant-mutant D-Ala-D-Lac peptide [6]. Another way is to modify the vancomycin molecule to redirect its target [7]. The polysaccharide backbone, however, often remains intact in the wild-type and antibiotic-resistant strains. We are, therefore, interested in development of new antibiotics targeting the glycosyltransferase reaction steps, especially the step of transglycosidase-catalyzed polysaccharide formation [8] (Fig. 1). We have overexpressed all the enzymes involved in the peptidogycan biosynthesis pathway and developed a fluorescence-based assay for evaluation of inhibitors. We use moenomycin, a known transglycosidase inhibitor [9], as a control and synthesized a fluorescently labeled uridine $5^{\prime}$-diphosphate (UDP)-muramylpeptide containing the terminal D-Ala-D-Lac sequence for use in discovering new transglycosidase inhibitors effective against vancomycin-resistant strains (Fig. 1). In addition, we have studied the mechanisms of glycosyltransferases and sulfotransferases and have developed new novel inhibitors to target these enzymes as a part of our efforts to develop new therapeutic strategies for the treatment of infectious and inflammatory diseases (Fig. 2). 


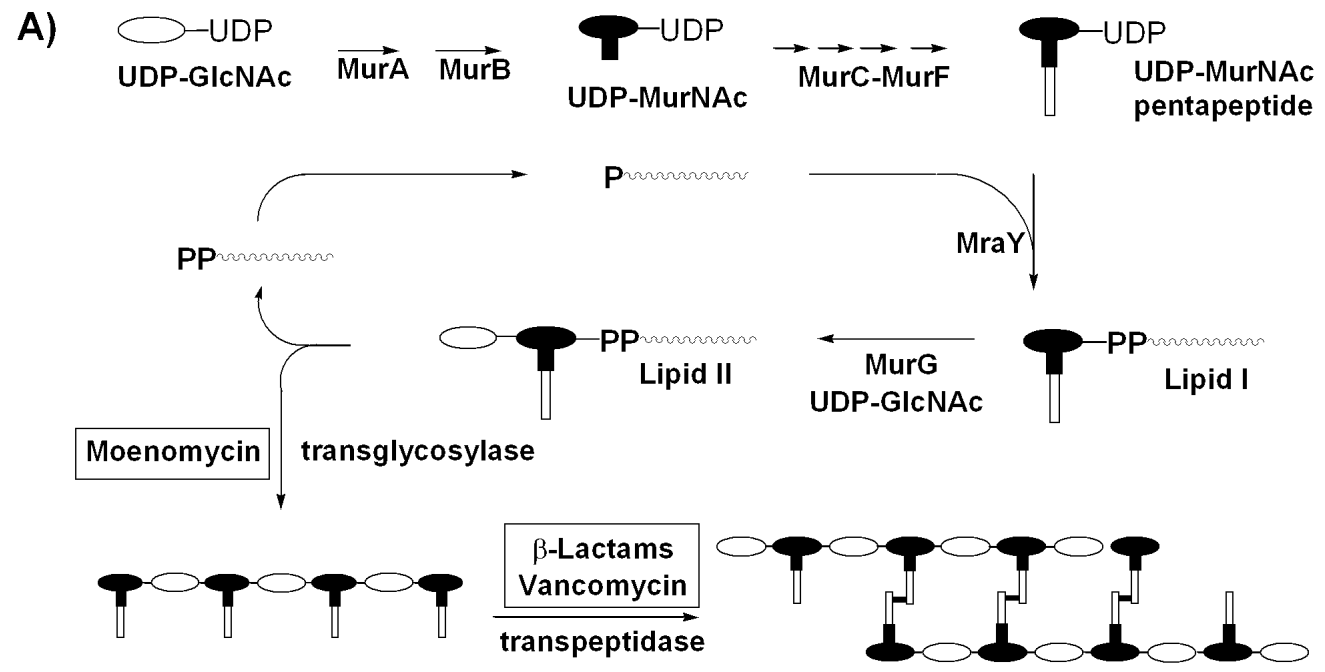

B)
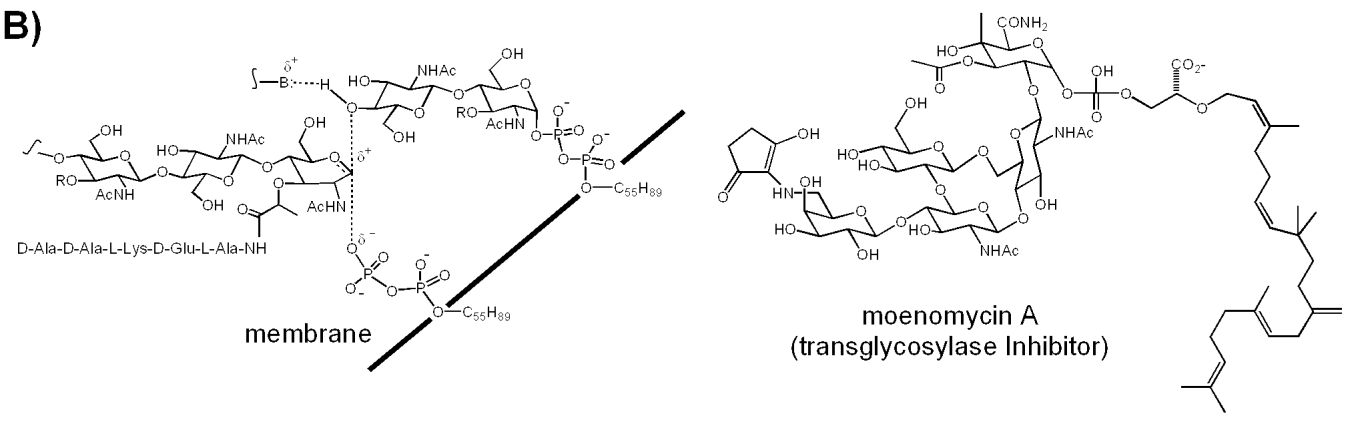

C)
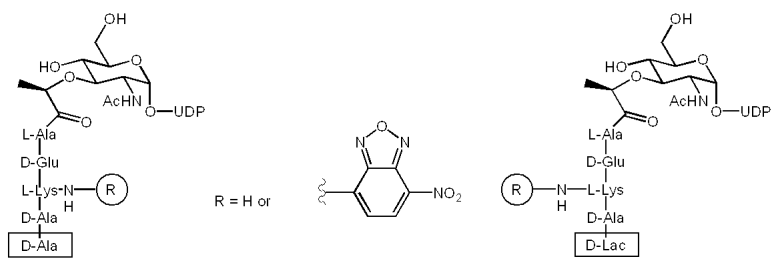

Fig. 1 Discovery of new antibiotics that target the glycosyltransfer enzymes (MurG and transglycosidase) involved in the biosynthesis of peptidoglycan. (A) The peptidoglycan synthesis pathway. (B) Proposed mechanism of the transglycosidase reaction and the structure of moenomycin A, an inhibitor of the enzyme. (C) UDP-muramylpeptides were prepared and used as substrates for the inhibition analysis of potential antibiotics active against vancomycin-resistant strains. 

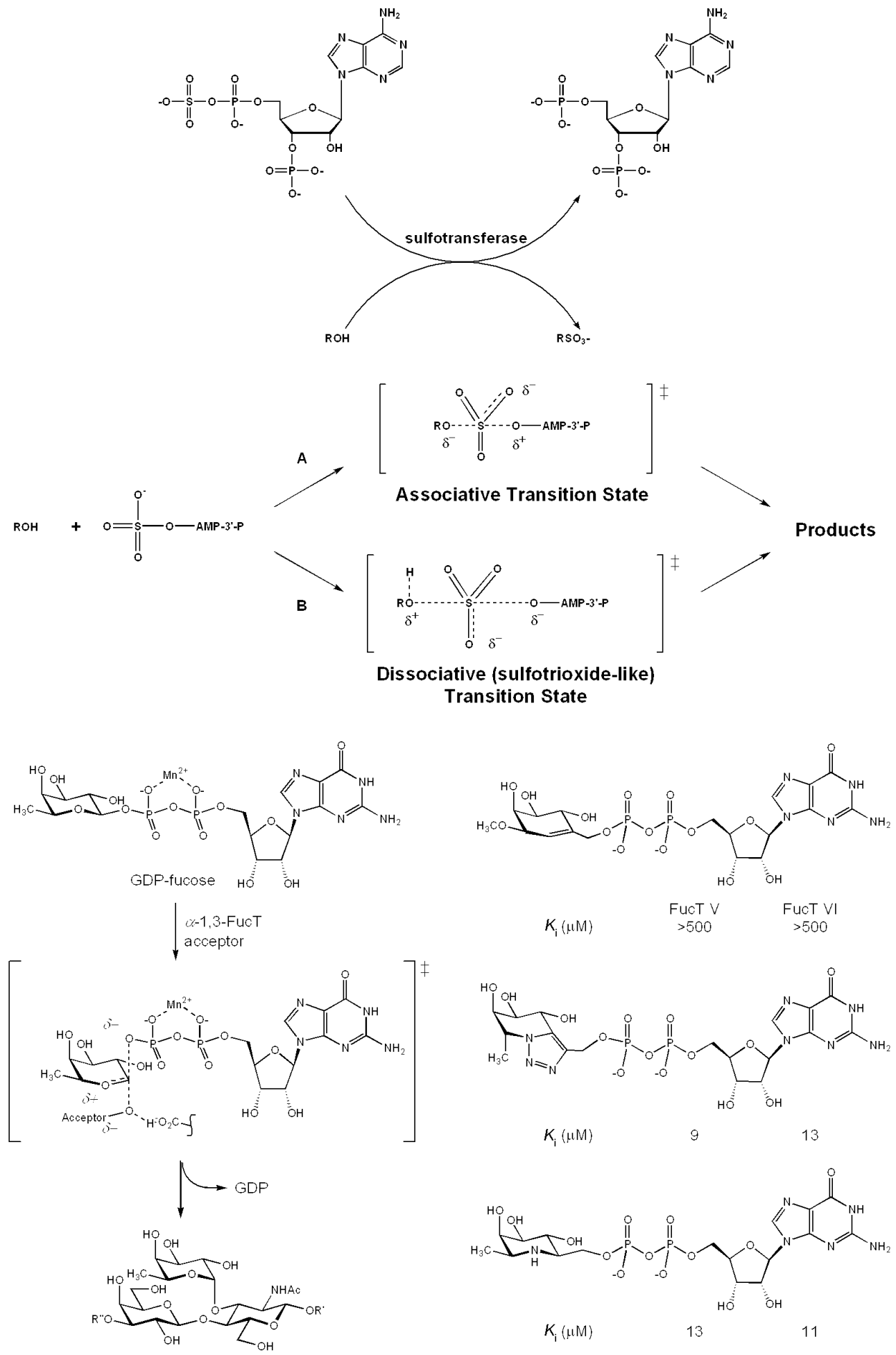

Fig. 2 Mechanisms of sulfotransferases and glycosyltransferases. Indicated are the dissociative mechanisms of arylsulfotransferase and the proposed transition states of fucosyltransferase-catalyzed reaction, as well as the transition-state analog inhibitors. 


\section{RNA-SMALL MOLECULE INTERACTION}

Another approach to develop novel antibiotics is to target the bacterial genome, especially RNA, as bacterial RNA is in the cytoplasm and accessible to small molecules [10]. In addition, with greater understanding of the genome from different species, identification of unique RNA sequences for development of sequence-specific inhibitors is becoming a new area for research in drug discovery. We have studied the molecular recognition of aminoglycoside antibiotics-RNA interaction [10] and have discovered that the $\beta$-hydroxyamine motif commonly found in aminoglycoside antibiotics plays a key role in interaction with the phosphodiester and the Hoogsteen face of the guanine residue in RNA (Fig. 3). We have been using this motif to design new antibiotics to target the bacterial 16S RNA A-site-a decoding sequence associated with protein biosynthesis. Aminoglycosides were prepared (Fig. 4) and used for the binding study using surface plasmon resonance. Small molecules with high affinity were further evaluated in the translation inhibition assay and antibiotic assay. Through this study, we have discovered a divalent neamine, which targets this sequence with $K_{\mathrm{d}}=40 \mathrm{nM}$, and the dimer also inhibits a

A)

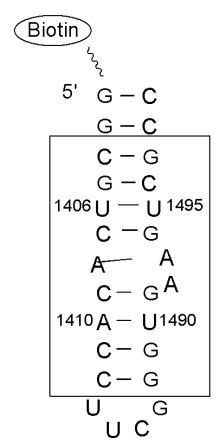

bacterial 16S RNA
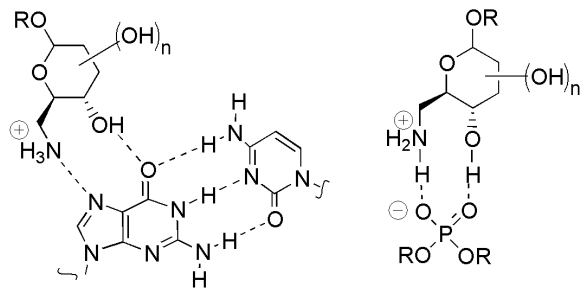

A-site

B)

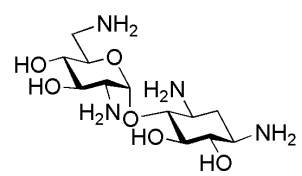

GC base Pair

phosphodiester

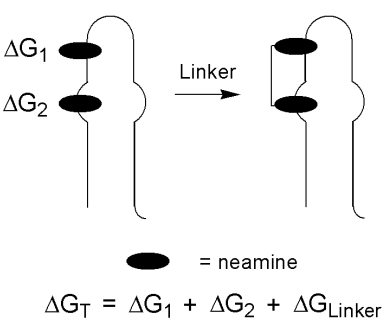

C)

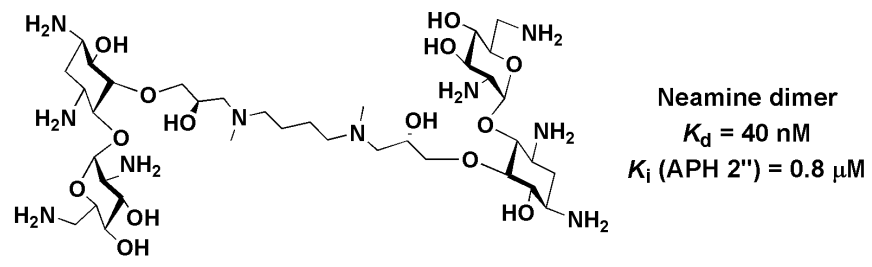

Fig. 3 Development of new antibiotics targeting bacterial 16S RNA A-site. (A) Biotinylated 16S A-site model was prepared for the binding analysis of small molecule-RNA interaction using surface plasmon resonance. The $\beta$-hydroxyamine motif commonly found in aminoglycoside antibiotics often interacts with the phosphodiester linkage and the Hoogsteen face of the G residue in RNA. (B) The binding analysis shows that the A-site contains two independent binding sites for neamine, with $K_{\mathrm{d}}=10 \mu \mathrm{M}$ for each site. Also shown is the energetic analysis of a neamine dimer with a linker. (C) A neamine dimer was found to bind the A-site with $K_{\mathrm{d}}=40 \mathrm{nM}$ and inhibit a resistance-causing enzyme $\left(\mathrm{APH}_{2}{ }^{\prime \prime}\right)$ with $K_{\mathrm{i}}-0.8 \mu \mathrm{M}$. 

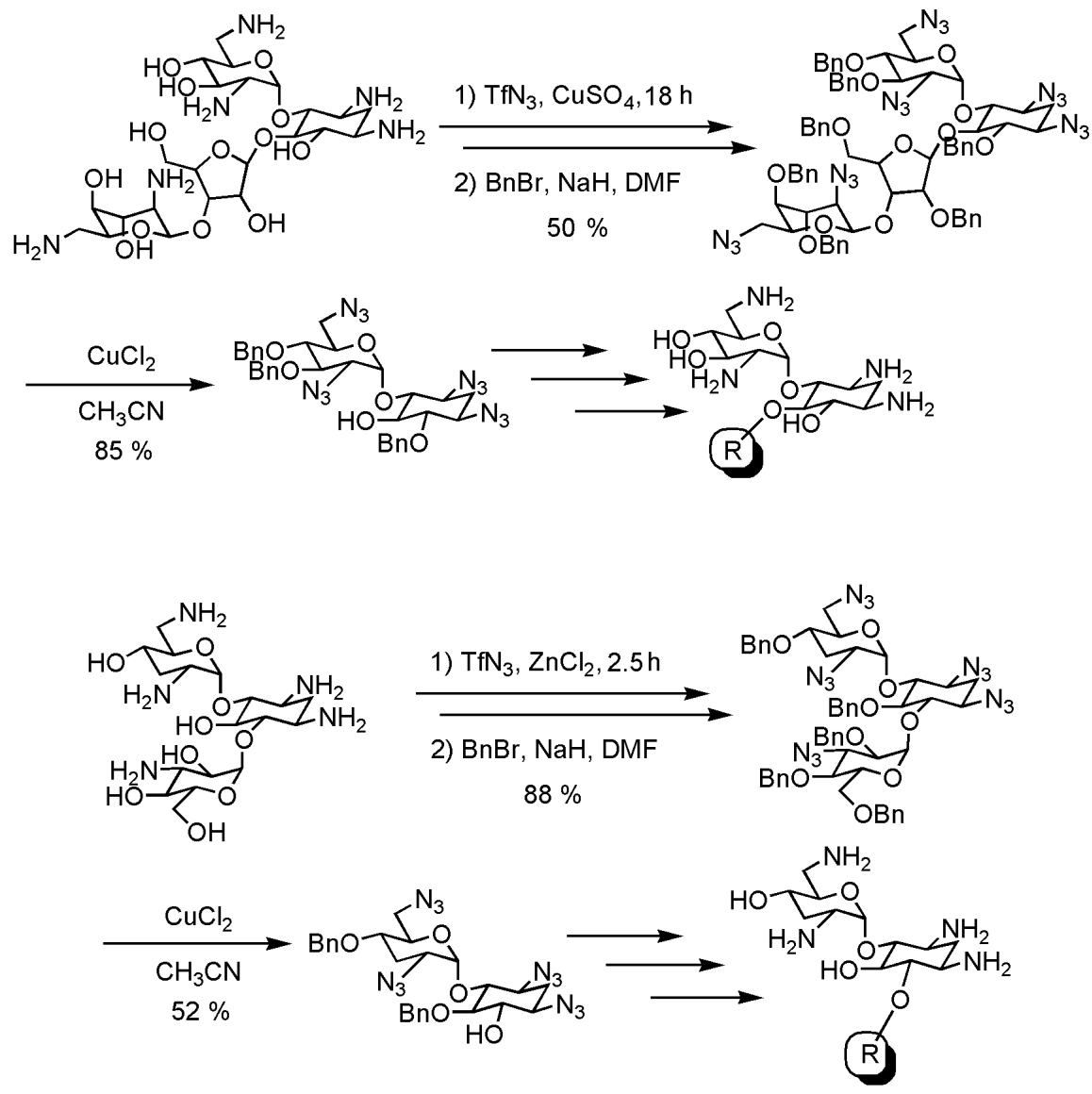

Fig. 4 Metal-catalyzed diazotransfer as a key step for the preparation of aminoglycoside derivatives.

phosphoryltransferase $\left(K_{\mathrm{i}}=0.8 \mu \mathrm{M}\right)$ involved in the modification of aminoglycosides to cause drug resistance [12]. This discovery has led to a new direction in antibiotic development. We have also extended this research to facilitate the discovery of new oligosaccharides or aminoglycosides to target proteins or RNA sequence specifically, using the programmable one-pot synthesis strategy [13] to prepare a large number of individual saccharides attached noncovalently to the surface of microtiter plate wells, and the arrays have been used to identify new saccharides as new protein ligands or as new antibiotics and antiviral agents (Fig. 5). 

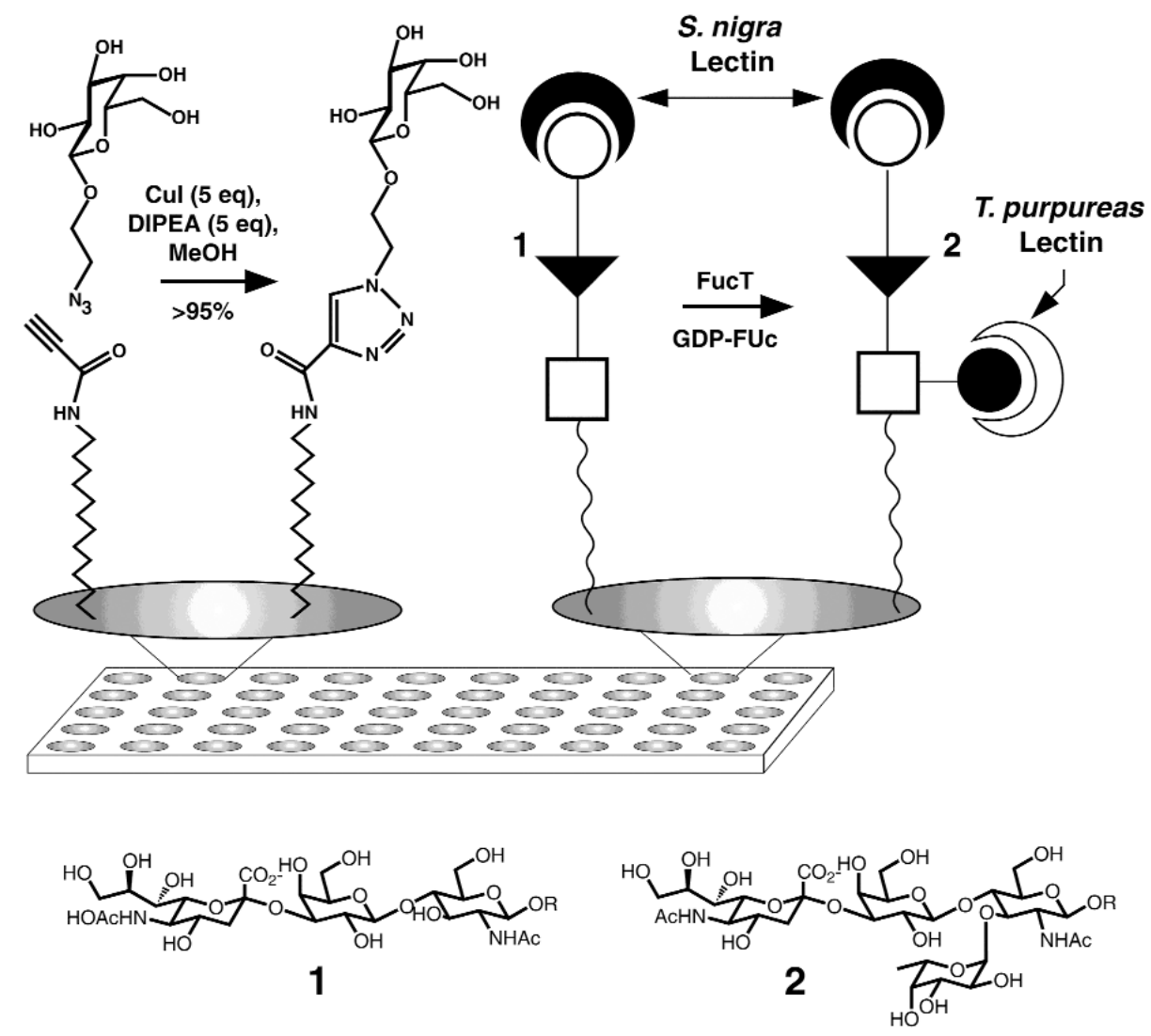

Fig. 5 Synthesis of sugar arrays in microtiter plates for screening. The $C_{13}-C_{15}$ saturated hydrocarbon spacer is found to be optimal for the noncovalent attachment of saccharides to the plate surface.

\section{CONCLUSION}

The carbohydrate-based approach to the development of new antibiotics to combat drug resistance as described here provides a new direction in drug discovery. The programmable one-pot synthesis technology has enabled the diversity-oriented synthesis of oligosaccharides and aminoglycosides as individual molecules or as arrays for high-throughput screening.

\section{REFERENCES}

1. (a) J. Davies. Science 264, 375 (1994); (b) F. A. Waldvogel. N. Engl. J. Med. 340, 556 (1999).

2. (a) D. H. Williams and B. Bardsley. Angew. Chem., Int. Ed. 38, 1172 (1999); (b) C. T. Walsh. Science 284, 442 (1999); (c) R. Nagarajan. Antimicrob. Agents Chemother. 35, 605 (1991).

3. D. T. W. Chu, J. J. Plattner, L. Katz. J. Med. Chem. 39, 3852 (1996).

4. I. A. Kozlov, Y. Xu, X. Huang, L. Lee, P. S. Sears, S. Mao, K. D. Janda, C.-H. Wong. ChemBiochem 2, 741 (2001).

5. (a) O. Schneewind, A. Fowler, K. F. Faull. Science 268, 103 (1995); (b) V. L. Healy, I. A. Lessard, D. I. Roper, J. R. Know, C. T. Walsh. Chem. Biol. 7, R109 (2000).

6. J. Rao, L. Yan, J. Lahiri, G. M. Whitesides, R. M. Weis, H. S. Warren. Chem. Biol. 6, 353 (1999).

7. M. Ge, Z. Chen, H. R. Onishi, J. Kohler, L. L. Silver, R. Kerns, S. Fukuzawa, C. Thomson, D. Kahne. Science 284, 507 (1999). 
8. S. Ha, E. Chang, M.-C. Lo, H. Men, P. Park, M. Ge, S. Walker. J. Am. Chem. Soc. 121, 8415 (1999).

9. S. Riedel, A. Donnerstag, L. Hennig, P. Welzel. Tetrahedron 55, 1921 (1999).

10. (a) D. J. Ecker, R. H. Griffey. DDT 4, 420 (1999); (b) T. Hermann. Angew. Chem., Int. Ed. 39, 1890 (2000).

11. (a) P. Sears and C.-H. Wong. Angew. Chem., Int. Ed. 38, 2300 (1999); (b) M.-P. MingeotLeclercq, Y. Glupczynski, P. M. Tulkens. Antimicrob. Agents Chemother. 43, 727 (1999).

12. S. J. Sucheck, A. L. Wong, K. M. Koeller, D. D Boehr, K.-A. Draker, P. Sears, G. D. Wright, C.-H. Wong. J. Am. Chem. Soc. 122, 5230 (2000).

13. Z. Zhang, I. R. Ollmann, X.-S. Ye, R. Wischnat, T. Daasov, C.-H. Wong. J. Am. Chem. Soc. 121, 734 (1999). 